University of Nebraska - Lincoln

DigitalCommons@University of Nebraska - Lincoln

Uniformed Services University of the Health

Sciences

U.S. Department of Defense

2011

\title{
Educating Clerkship Students in the Era of Resident Duty Hour Restrictions
}

L. James Nixon

University of Minnesota, nixon007@umn.edu

Meenakshy Aiyer

University of Illinois College of Medicine at Peoria

Steven Durning

Uniformed Services University of the Health Sciences

Chris Gouveia

University of California - San Francisco

Jennifer R. Kogan

University of Pennsylvania School of Medicine

See next page for additional authors

Follow this and additional works at: https://digitalcommons.unl.edu/usuhs

Part of the Medicine and Health Sciences Commons

Nixon, L. James; Aiyer, Meenakshy; Durning, Steven; Gouveia, Chris; Kogan, Jennifer R.; Lang, Valerie J.; Cate, Olle ten; and Hauer, Karen E., "Educating Clerkship Students in the Era of Resident Duty Hour Restrictions" (2011). Uniformed Services University of the Health Sciences. 29.

https://digitalcommons.unl.edu/usuhs/29

This Article is brought to you for free and open access by the U.S. Department of Defense at DigitalCommons@University of Nebraska - Lincoln. It has been accepted for inclusion in Uniformed Services University of the Health Sciences by an authorized administrator of DigitalCommons@University of Nebraska Lincoln. 


\section{Authors}

L. James Nixon, Meenakshy Aiyer, Steven Durning, Chris Gouveia, Jennifer R. Kogan, Valerie J. Lang, Olle ten Cate, and Karen E. Hauer 


\title{
APM Perspectives
}

The Association of Professors of Medicine (APM) is the national organization of departments of internal medicine at the US medical schools and numerous affiliated teaching hospitals as represented by chairs and appointed leaders. As the official sponsor of The American Journal of Medicine, the association invites authors to publish commentaries on issues concerning academic internal medicine.

\section{Educating Clerkship Students in the Era of Resident Duty Hour Restrictions}

\author{
L. James Nixon, MD, ${ }^{a}$ Meenakshy Aiyer, MD, ${ }^{b}$ Steven Durning, MD, ${ }^{c}$ Chris Gouveia, ${ }^{d}$ Jennifer R. Kogan, MD, ${ }^{e}$ \\ Valerie J. Lang, MD, ${ }^{f}$ Olle ten Cate, PhD, ${ }^{g}$ Karen E. Hauer, MD $^{d}$ \\ ${ }^{a}$ University of Minnesota, Minneapolis; ${ }^{b}$ University of Illinois College of Medicine at Peoria; ${ }^{c}$ Uniformed Services University \\ of the Health Sciences, Bethesda, MD; ${ }^{d}$ University of California, San Francisco; ${ }^{e}$ University of Pennsylvania School of \\ Medicine, Philadelphia; ${ }^{f}$ University of Rochester School of Medicine, NY; ${ }^{g}$ University Medical Center Utrecht, The \\ Netherlands.
}

In 2003, the Accreditation Council for Graduate Medical Education defined for the first time an upper limit on the number of hours that residents can work. This initial reduction in work hours was made with the goal of improving patient safety, resident education, and resident well-being. ${ }^{1}$ Now, 8 years later, further duty hour restrictions are slated to take effect July 1, 2011. These new reductions are largely a response to growing public concern about the effects of sleep deprivation on residents. ${ }^{2}$ Educators and residents alike have mixed opinions about the effects of prior and future duty hour restrictions on resident education. ${ }^{3}$ The potential effects of resident duty hour restrictions on medical student education have received less attention.

Changes to graduate medical education have the potential to affect medical students' experiences and learning during their clerkships. With further duty hour restrictions imminent, now is the ideal time to learn from past duty hour restrictions and predict the likely effects of further resident duty hour restrictions on medical students. A forward-looking approach to the

\footnotetext{
Funding: None.

Conflict of Interest: None of the authors have any conflicts of interest associated with the work presented in this manuscript.

Authorship: All authors had access to the data and played a role in writing this manuscript.

Reprint requests should be addressed to L. James Nixon, MD, Associate Professor, University of Minnesota, 420 Delaware St SE, MMC 741, Minneapolis, MN 55455.

E-mail address: nixon007@umn.edu
}

new duty hours regulations allows medical educators to anticipate, and potentially prevent, negative effects while providing an opportunity to capitalize on potential benefits.

This article provides an overview of the current knowledge of the effects of duty hour restrictions on medical student education to guide educators in planning for consequences of further resident duty hour restrictions. A literature review was conducted using the MeSH subheadings "internship and residency" and "students, medical." These reviews were then combined with separate key word searches using the terms "duty hours" and "work hours." For articles deemed relevant to this topic, a related article search was conducted in PubMed and included articles' references were reviewed for relevance. The authors of this article include clerkship directors and medical educators, and many have published previously on this topic. This article was reviewed and endorsed by the Clerkship Directors in Internal Medicine Council in January 2011.

A narrative review of the literature demonstrates that most of the concerns regarding resident duty hour restrictions and medical student education address 4 key areas. These areas include teaching, discontinuity, team structure, and educational climate. Teaching concerns relate to residents' role as educator and rounds and the role of the attending. Discontinuity concerns relate to shift work and handoffs. For each area, we have sum- 
marized the current literature and interpreted the effects of these changes on medical student education. We also include best practices for teaching students in the context of these changes.
Concerns include less time available for teaching during rounds, less bedside teaching, ${ }^{11}$ and an overall decline in the medical student educational experience post-duty hour restrictions. ${ }^{10}$

Another concern is that duty hour restrictions may alter attending rounds' discussions to render them less educationally valuable for students. Internal medicine attending and resident physicians in a focus group study thought that rounds postduty hour restrictions emphasized the clinical and managerial aspects of rounding (focused on making clinical decisions) at the expense of teaching. ${ }^{19}$ Specifically, attendings were concerned that compressed time and emphasis on rounding efficiency had the potential to limit medical student opportunities to give traditional long patient presentations and learn from attendings at the bedside. Attendings did acknowledge that the increased focus of rounds on clinical care promoted opportunities to observe how learners process information in real time. ${ }^{19}$ This perception of less time for teaching is supported by a study that found $29 \%$ of rounding time was spent on educational activities in internal medicine pre-duty hour restrictions, ${ }^{20}$ whereas a separate study performed post-duty hour restrictions found that $9 \%$ of time during internal medicine rounds was spent on educational activities. $^{21}$

Attendings' predominantly negative views of duty hour restrictions contrast with the majority of studies evaluating student perspectives. Students generally perceived little impact of duty hour restrictions on attending availability or quality of teaching. ${ }^{7,12,13,16}$ Specifically, students' ratings of attendings' interest, skill, and availability, as well as the quality and quantity of their feedback, did not significantly change. ${ }^{13}$ Similarly, duty hour restrictions did not translate into decreased teaching by attendings or decline in teaching quality or feedback provided in the medicine or surgery clerkships. ${ }^{7}$ In addition, there was no change in the proportion of time medicine clerkship students spent on attending rounds or the ratings of their educational value pre- to post-duty hour restrictions. ${ }^{12}$ Notable exceptions to these general trends include a decline in avail-

ability of faculty post-duty hour restrictions for surgical restrictions will threaten their attending role. ${ }^{10,11,19}$

\section{Rounds and the Role of the Attending Physician}

In teaching hospitals, attending rounds are a core activity whereby students learn the practice of medicine and are the primary means for information exchange in the provision of patient care. Learners on the clinical wards value faculty who are available and interested in teaching. ${ }^{17,18}$ Faculty, especially if heavily involved with student teaching, tend to believe that duty hour 
services at one institution ${ }^{16}$ and a decline in pediatrics teaching at another. ${ }^{7}$

It is difficult to reconcile resident and attending perceptions with student perceptions of duty hour restrictions. We were unable to locate any side-by-side comparison studies exploring these differences. It is possible that resident and attending effort or other structural changes have protected students from these effects. Alternatively, different perspectives may stem from methodological difficulties in measuring differences, particularly without randomized controlled trials.

\section{DISCONTINUITY}

\section{Shift Work}

Medical educators have expressed concern that increases in shift work will decrease student continuity with their team and patients. This discontinuity could erode students' sense of ownership for their patients, thereby impairing their professional development. ${ }^{11,22}$ Similarly, excessive shift work could erode a learner's work ethic and sense of responsibility to patients. ${ }^{23}$ The actual impact of duty hour restrictions on student continuity with patients has been mixed. Key clinical faculty have worried that student ability to follow a patient throughout a hospitalization has worsened post-duty hour restrictions, ${ }^{10}$ whereas clerkship directors think that student ability to follow patients is unchanged. ${ }^{11}$

From the student perspective, despite reporting fewer hours worked at one institution, they noted no change in the quality of their relationships with patients pre- to post-duty hour restrictions. ${ }^{7}$ This finding may be partially explained by the lack of major structural change to the core medical student activities. The students worked fewer hours, but no changes were made to overnight call (absent pre- and post-duty hour restrictions) and no new experiences such as night float or day float were added. This observation may be transferable to other clerkships given the infrequency with which medicine clerkships have required overnight call both before and after duty hour restrictions. ${ }^{11}$ Most likely, required overnight call for medical students will continue to decline as residents do less overnight call. Whether students will start to have a role on night float or other teams outside of the traditional team remains unknown. The impact of overnight call on student learning is unclear, and students seem ambivalent about its value. ${ }^{24}$

\section{Handoffs}

In response to duty hour restrictions, many residency programs have implemented night float, day float, and other shift work models that can increase patient handoffs. ${ }^{25}$ Concurrent with these changes, handoffs have been estimated to increase by $11 \%$ to $40 \% .^{26,27} \mathrm{Al}-$ though transfers of care are becoming more prevalent, only $8 \%$ of medical schools have any curricula about handoffs. ${ }^{28}$ Although the impact of night float on patient care, resident health, and resident education has been examined, ${ }^{25}$ the implications for medical student learning and the overall clerkship experience are not clear. One study showed that "fresh" patients (ie, patients from the office or emergency department who have not been given a diagnosis to explain their clinical presentation) comprised just more than one half of student admissions and that the number of "fresh" patients a student admitted positively correlated with their score on the National Board of Medical Examiners' subject examination. ${ }^{29}$ Whether students will assume roles on night float or teams outside of the traditional team also is unknown, but the effects on their exposure to "fresh patients" will need to be considered.

\section{STRUCTURE: CHANGE IN TEAM MEMBERS}

As residents have fewer hours in which to perform their work and attending physicians have increasing responsibility, hospital leaders have attempted to alter work load on the teaching services through addition of faculty-only teams, non-physician practitioners, or day float/night float residents. ${ }^{30,31}$ These structural and workforce changes can affect medical student education by freeing up resident and attending time for teaching. ${ }^{32,33}$ In fact, some of the programs observed positive or no effects of duty hour restrictions on student education when they used non-physician practitioners to offset the work of the teaching teams. ${ }^{12,16}$

Structural changes also may alter students' clinical responsibilities. In one example, duty hour restrictions shifted activities of minimal educational value to medical students on medicine and surgery clerkships ${ }^{16} \mathrm{Stu}-$ dents subsequently perceived less time available for independent study and learning. The addition of 24hour hospitalist coverage for a pediatrics service at the same hospital, however, ameliorated this perceived influx of "scutwork." 16

\section{EDUCATIONAL CLIMATE: CHANGE IN STUDENT DUTY HOURS}

Little has been written specifically addressing the educational climate for students, but studies have found no significant difference in the time spent on or the quality of student learning activities pre- to post-duty hour restrictions. The current duty hour restrictions specifically apply to residents, not students. However, a survey of deans of the Liaison Committee on Medical Education-accredited US medical schools found that most schools have a written policy restricting student duty hours. ${ }^{34}$ There was not consensus across schools on what those regulations should entail, and only one half 
Table Managing Duty Hour Restrictions for the Benefit of Student Education

\begin{tabular}{|c|c|}
\hline Key Areas & Best Practices \\
\hline Teaching & $\begin{array}{l}\text { Develop attendings' skills during rounds: } \\
\text { Concise, targeted teaching, such as microskills model }{ }^{35} \\
\text { Observe learners process information in real time and provide feedback } \\
\text { Teach in relation to real-time patient assessment and medical decision-making } \\
\text { Develop attendings' skills for outside of rounds or separate teaching attending: } \\
\text { In-depth bedside teaching of physical examination and communication skills } \\
\text { Review and critique comprehensive presentations and associated medical decision-making } \\
\text { Develop students' skills: } \\
\text { Direct patient case presentations during rounds, such as SNAPPS } \\
\text { Reflect on performance and sharing learning goals } \\
\text { Develop residents' skills: } \\
\text { Incorporate students into a clinical team } \\
\text { Best practices for teaching } \\
\text { Include developmentally appropriate experiences for learners } \\
\text { Include competencies and milestones to mark learner progress }\end{array}$ \\
\hline Discontinuity & $\begin{array}{l}\text { Maximize opportunities to evaluate "fresh" patients or simulate "fresh" patients: } \\
\text { Night float or other rotations where more new patients are admitted } \\
\text { "Blind" evaluations of patients before reviewing night-float's admit-note } \\
\text { Live or computer-based simulations } \\
\text { Teach students best practices for giving and receiving hand-offs } \\
\text { Improve instruction in patient safety and quality improvement as it relates to hand-offs }\end{array}$ \\
\hline Team structure & $\begin{array}{l}\text { Use hospitalist services and non-physician practitioner to redistribute workload and maximize educational } \\
\text { value of ward experience } \\
\text { Clearly define the roles and responsibilities of all team members } \\
\text { Consider new structures for training that are less dependent on residents }\end{array}$ \\
\hline $\begin{array}{l}\text { Educational } \\
\text { climate }\end{array}$ & Clearly define student work hours \\
\hline
\end{tabular}

climate

of respondents supported the Accreditation Council for Graduate Medical Education duty hour regulations for students. Although deans thought that restriction of student hours enhances student well-being, most believed its impact on patient care, student evaluation, direct time with patients, and the student-resident team relationship would be neutral. As discussions proceed regarding the impact of resident duty hour restrictions on medical student education, consideration will need to be given to student duty hour regulations, particularly if regulations and policies differ. It is unclear whether these changes in student duty hours have resulted in an improvement in student quality of life, but residents with similar changes have thought that duty hour restrictions helped them live a more balanced life. ${ }^{9}$

\section{BEST PRACTICES FOR MANAGING DUTY HOUR RESTRICTIONS FOR THE BENEFIT OF STUDENT EDUCATION}

\section{Teaching}

In light of the greater time pressure during rounds, models for improving teaching efficiency and effective- ness should be adopted (Table). Concise, targeted teaching is expected in the ambulatory setting, and these same skills will need to be adapted to the inpatient teaching arena. Potentially successful models include the microskills model ${ }^{35}$ and Summarize, Narrow, Analyze, Probe, Plan, Select (SNAPPS), ${ }^{36}$ which emphasize concise and directed patient case presentations. Teaching learners to reflect on their performance and share their learning goals will allow attendings to make the most of teachable moments. Because attending rounds post-duty hour restrictions have a greater focus on clinical decision-making, attendings should capitalize on this opportunity to observe learners process information and teach skills in patient assessment and medical decision-making.

High demands for rapid delivery of patient care necessitate shifting some educational activities out of work rounds into dedicated teaching venues. For instance, comprehensive student oral presentations may occur as a part of the clerkship teaching structure and separate from the teams. Attendings may choose to dedicate individual time later in the day to provide students bedside teaching of physical examination and communication skills or focus on presentations and medical decision-making. Models that rely on the at- 
tending physician for offloading resident work will likely require a separate teaching physician to fulfill these student teaching roles.

Faculty development should address effective methods of teaching in this environment. Residents should be taught best practices for teaching and incorporating students into a clinical team post-duty hour restrictions.

\section{Discontinuity}

Shift work and handoffs are here to stay, so maximizing learning in this environment is essential. Students should have opportunities to evaluate fresh patients; student rotations on night float teams or other teams that routinely admit new patients may facilitate student "first contact" with patients. Interventions to improve the "freshness" of handoff patients could include having students perform evaluations of patients before reviewing the night float team's admissions note or patient chart. Computer-based simulations are another option for learning the approach to an undifferentiated patient. Students need to learn strategies for both giving and receiving handoffs; students also should be given the opportunity to understand how they relate to the broader issues of patient safety and quality improvement.

\section{Structure}

A health care system's response to duty hour restrictions should include a proactive and prospective approach. Those programs that had more positive response to prior duty hour restrictions anticipated the coming change and designed systems to maximize education in a new environment. Systems can maximize learning for students by the addition of hospitalist services and non-physician practitioners that can help redistribute workload to maximize its educational value. Direct costs are associated with the addition of these services, but also there is evidence that such changes can be cost neutral. ${ }^{37}$

New care delivery models will require clear delineation of the roles and responsibilities of all team members, including medical students. Lack of role delineation and assumption of too much responsibility by non-physician practitioners have been cited as potential challenges of using non-physician practitioners in residency training. ${ }^{38}$ It is important to define the student role in patient care, outline resident teaching responsibilities, and articulate the relationships between the medical students and non-physician practitioners within the health care team to ensure a positive learning climate and educational outcomes. Student participation on multidisciplinary teams including non-physician practitioners creates the opportunity to highlight interprofessional education and enhance medical student education post-duty hour restrictions.

\section{Educational Climate}

Individual schools should define clearly the duty hour limitations for students on clinical clerkships. There should be consideration for a national consensus defining these work hours across Liaison Committee on Medical Education-accredited schools.

\section{LIMITATIONS}

Our observations and recommendations should be taken with many caveats. We recognize duty hour restrictions are not the only changes in the current dynamic clinical environment with the potential to affect student education. Also, most of the published studies on which we are basing our recommendations were observational and single institution, representing only a few specialties; most outcomes were attitudes, perceptions, and satisfaction versus more robust outcomes such as performance.

\section{CONCLUSIONS AND RECOMMENDATIONS}

There is ongoing pressure from the evolving clinical and educational environment on our traditional Flexnerian apprenticeship model for training medical students on their inpatient clerkships. Adaptation of this traditional model may no longer be sufficient to train students to practice medicine in the 21 st century. It may be time to consider whether students would be better served by a new structure of undergraduate training that is less dependent on residents. This structure may require increased use of simulation with subtle modification of the current structure or a more significant shift to models such as longitudinal integrated clerkships. ${ }^{39}$ Regardless, the ideal model should include developmentally appropriate experiences for learners and competencies and milestones to mark learner progress to ensure that, despite the potential for fewer hours spent in the hospital, our learners are still achieving the same outcome. $^{40}$

\section{References}

1. Steinbrook R. The debate over residents' work hours. $N$ Engl J Med. 2002;347:1296-1302.

2. Ulmer CWD, Johns M, eds. Resident Duty Hours: Enhancing Sleep, Supervision, and Safety. Washington, DC: National Academies Press; 2008.

3. Drolet BC, Spalluto LB, Fischer SA. Residents' perspectives on ACGME regulation of supervision and duty hours - a national survey. N Engl J Med. 2010;363:e34.

4. Boex JR, Leahy PJ. Understanding residents' work: moving beyond counting hours to assessing educational value. Acad Med. 2003;78:939-944.

5. O'Sullivan PS, Weinberg E, Boll A, Nelson TR. Students educational activities during clerkship. Acad Med. 1997;72:308-313.

6. 2010 Medical School Graduation Questionnaire. Final All Schools Report. Association of American Medical Colleges, 2010. Available at: https://www.aamc.org/download/140716/data/ 2010_gq_all_schools.pdf. Accessed December 15, 2010. 
7. Nixon LJ, Benson BJ, Rogers T, et al. Effects of Accreditation Council for Graduate Medical Education work restrictions on medical student experience. J Gen Intern Med. 2007;22:937-941.

8. Zahn CM, Dunlow SG, Alvero R, et al. Too little time to teach? Medical student education and the resident work-hour restriction. Mil Med. 2007;172:1053-1057.

9. Lin GA, Beck DC, Garbutt JM. Residents' perceptions of the effects of work hour limitations at a large teaching hospital. Acad Med. 2006;81:63-67.

10. Reed DA. Impact of duty hour regulations on medical students' education: views of key clinical faculty. J Gen Intern Med. 2008;23:1084-1089.

11. Kogan JR. The impact of resident duty hours reform on the internal medicine core clerkship: results from the clerkship directors in internal medicine. Acad Med. 2006;81:1038-1044.

12. Kogan JR, Bellini LM, Shea JA. The impact of resident duty hour reform on a medicine core clerkship. Acad Med. 2004;79: S58-S61.

13. Jagsi R, Shapiro J, Weinstein DF. Perceived impact of resident work hour limitations on medical student clerkships: a survey study. Acad Med. 2005;80:752-757.

14. Arora VM. Effect of student duty hours policy on teaching and satisfaction of 3rd year medical students. Am J Med. 2006;119: 1089-1095.

15. Brasher AE. Medical students' perceptions of resident teaching: have duty hours regulations had an impact? Ann Surg. 2005;242: 548-553.

16. White CB. Multidimensional effects of the 80 -hour work week at the University of Michigan Medical School. Acad Med. 2006; 81:57-62.

17. Sutkin G, Waner E, Harris I, Schiffer R. What makes a good clinical teacher in medicine? A review of the literature. Acad Med. 2008;83:452-466.

18. Ullian JA, Bland CJ, Simpson DE. An alternative approach to defining the role of the clinical teacher. Acad Med. 1994;69:832838.

19. Harrison R, Allen E. Teaching internal medicine residents in the new era: inpatient attending with duty hour regulations. $J$ Gen Intern Med. 2006;21:447-452.

20. Elliot DL, Hickam DH. Attending rounds on in-patient units: differences between medical and non-medical services. Med Educ. 1993;27:503-508.

21. Priest JR, Bereknyei S, Hooper K, Braddock CH. Relationships of the location and content of rounds to specialty, institution, patient-census, and team size. PLoS One. 2010;5:e11246.

22. Schwartz A, Pappas C, Bashook PG, et al. Conceptual frameworks in the study of duty hours changes in graduate medical education: a review. Acad Med. 2011;86:18-29.

23. Woodrow SI, Segouin C, Armbruster J, et al. Duty hours reforms in the United States, France, and Canada: is it time to refocus our attention on education? Acad Med. 2006;81:1045-1051.

24. Corriere M, Denton D. Overnight call: a survey of medical student experiences, attitudes and skills. Presented at Clerkship Directors in Internal Medicine National Meeting 2010. Available at: http://www.im.org/Meetings/Past/2010/AIMW10/Presentations/ Documents/2010\%20CDIM\%20National\%20Meeting/PSIII_ Corriere.pdf. Accessed January 21, 2011.
25. Reed DA, Fletcher KE, Arora VM. Systematic review: association of shift length, protected sleep time, and night float with patient care, residents' health and education. Ann Intern Med. 2010;153:829-842.

26. Vidyarthi AR, Arora V, Schnipper JL, Wall SD, Wachter RM. Managing discontinuity in academic medical centers: strategies for a safe and effective resident sign-out. J Hosp Med. 2006;1: 257-266.

27. Horwitz LI, Krumholz HM, Green ML, Huot SJ. Transfers of patient care between staff on internal medicine wards. Arch Intern Med. 2006;166:1173-1177.

28. Solet DJ, Norvell JM, Rutan GH, Frankel RM. Lost in translation: challenges and opportunities in physician-to-physician communication during patient handoffs. Acad Med. 2005;80: 1094-1099.

29. Lang VJ, Mooney CJ, O'Connor AB, Bordley DR, Lurie SJ. Association between handoff patients and subject exam performance in medicine clerkship students. J Gen Intern Med. 2009; 24:1018-1022. Epub 2009 Jul 5.

30. Weinstein DF. Duty hours for resident physicians-tough choices for teaching hospitals. $N$ Engl J Med. 2002;347:12751278 .

31. Gordon CR, Axelrad A, Alexander JB, Dellinger RP, Ross SE. Care of critically ill surgical patients using the 80-hour Accreditation Council of Graduate Medical Education work-week guidelines: a survey of current strategies. Am Surg. 2006;72(6): 497-499.

32. Cawley JF, Hooker RS. The effects of resident work hour restrictions on physician assistant hospital utilization. J Phys Asst Educ. 2006;17:41-43.

33. Cooper RA. New directions for nurse practitioners and physician assistants in the era of physician shortages. Acad Med. 2007;82: 827-828.

34. Friedman E, Karani R, Fallar R. Regulation of medical student work hours: a national survey of deans. Acad Med. 2011;86: 30-33.

35. Neher JO, Gordon KC, Meyer B, Stephens N. A five-step "microskills" model of clinical teaching. Clin Teach. 1992;5:419424.

36. Wolpaw TM, Wolpaw DR, Papp KK. SNAPPS: a learner-centered model for outpatient education. Acad Med. 2003;78:893898.

37. Lundberg S, Balingit $\mathrm{P}$, Wali S, Cope D. Cost-effectiveness of a hospitalist service in a public teaching hospital. Acad Med. 2010; 85:1312-1315.

38. Mathur M, Rampersad A, Howard K, Goldman GM. Physician assistants as physician extenders in the pediatric intensive care unit setting - a 5-year experience. Pediatr Crit Care Med. 2005; 6:14-19.

39. Norris TE, Schaad DC, DeWitt D, et al. Longitudinal integrated clerkships for medical students: an innovation adopted by medical schools in Australia, Canada, South Africa, and the United States. Acad Med. 2009;84:902-907.

40. Carracio C, Benson BJ, Nixon LJ, Derstine P. From the educational bench to the clinical bedside: translating the Dreyfus developmental model to the learning of clinical skills. Acad Med. 2008;83:761-767. 